\title{
Minimum size and positioning of imaging field for CBCT scans of impacted maxillary canines
}

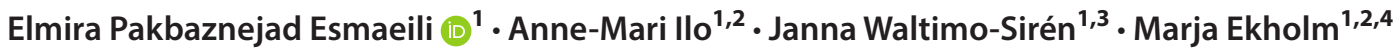

Received: 8 November 2018 / Accepted: 4 April 2019/Published online: 24 June 2019

(c) The Author(s) 2019

\begin{abstract}
Objectives In children and adolescents, cone-beam computed tomography (CBCT) is frequently used for localization of unerupted or impacted teeth in the anterior maxilla. CBCT causes a higher radiation dose than conventional intraoral and panoramic imaging. The objective was to analyze the location of impacted canines in a three-dimensional coordinate and thereby optimize the CBCT field-of-view (FOV), for radiation dose reduction.

Materials and methods Location of 50 impacted maxillary canines of children under 17 years was retrospectively evaluated from CBCT scans. The minimum and maximum distances of any part of the right- and left-side canines to three anatomic reference planes were measured to assess the adequate size and position of a cylindrical image volume.

Results A cylinder sized 39.0 (diameter) $\times 33.2$ (height) $\mathrm{mm}$, with its top situated $13.8 \mathrm{~mm}$ above the hard palate, its medial edge $8.4 \mathrm{~mm}$ across the midline, and anterior edge $2.5 \mathrm{~mm}$ in front of the labial surface of maxillary central incisors fitted all the analyzed canines.

Conclusions In this sample, the FOV required for imaging maxillary impacted canines was smaller than the smallest FOV offered by common CBCT devices. We encourage development of indication-specific CBCT imaging programs and aids to facilitate optimum patient positioning.

Clinical relevance An impacted maxillary canine is a common dental problem and a frequent indication for 3D imaging particularly in growing individuals. This article focuses on the optimization of CBCT of impacted canines. Our recommendation of a reduced FOV promotes radiation safety.
\end{abstract}

Keywords Cone-beam computed tomography $\cdot$ Radiation protection $\cdot$ Child $\cdot$ Cuspid $\cdot$ Tooth $\cdot$ Impacted

\section{Introduction}

Cone-beam computed tomography (CBCT) is a threedimensional (3D) radiographic method used in dentistry mainly for imaging of teeth, jaws, and mid-facial bony structures [1]. In general, CBCTs are indicated when 2D

ElmiraPakbaznejad Esmaeili

elmira.pakbaznejad@helsinki.fi

1 Department of Oral and Maxillofacial Diseases, University of Helsinki, P.O. Box 41, 00014 Helsinki, Finland

2 Institute of Dentistry, University of Turku, Turku, Finland

3 Department of Oral and Maxillofacial Diseases, Helsinki University Hospital, Helsinki, Finland

4 South West Finland Imaging Centre, Turku University Hospital, Turku, Finland radiographs and other alternative techniques using lower amount of radiation are not sufficient for patient management. Individual needs of the patients, based on history of the disease and clinical findings, determine the necessity of CBCT scans. When indicated, CBCTs are applied for example for orthodontic, endodontic, and surgical reasons, for assessment of periapical diseases, bone pathologies, dento-alveolar and facial trauma, and temporomandibular joints [1]. Localization of maxillary unerupted or impacted anterior teeth forms a common indication for CBCT in adolescents [2, 3]. Compared to two-dimensional (2D) imaging methods, CBCT is more precise and accurate in localizing unerupted maxillary canines and detection of root resorption in adjacent teeth [4-7], but it exposes the patient to a higher radiation dose. Therefore, according to Wriedt et al., CBCT with a small field-of-view (FOV) would be justified when canines incline more than $30^{\circ}$ from a vertical position, and there is suspicion of resorption of adjacent teeth, 
or when the apex area of the canine is not well observable on dental panoramic tomogram (DPT) [8].

When imaging children and adolescents with CBCT, it is particularly important to keep the radiation dose at a minimum, because of their especial vulnerability to harmful effects of ionizing radiation. Creating a balance between factors influencing the patient dose and image quality is recommended patient, age, and indication specifically by the European DIMITRA project (dentomaxillofacial pediatric imaging: an investigation toward low-dose radiation-induced risk) [9]. The dose received by the patient is determined by the exposure parameters, filtration, type of detector, voxel size, number of projections, shielding devices, and, importantly, size of FOV [1].

The size of FOV is usually displayed as $D \varnothing \times H \mathrm{~cm}$, where $D$ is the diameter and $H$ is the height of the cylindrical volume. Different devices have different choices for FOV, and the imaging field may cover a few teeth, jaws, or even both midface and mandible at the same time. In general, the smaller the FOV, the lower the radiation dose. Therefore, one should apply the minimum size of FOV, only covering the structures of interest [1]. Today, a lot of effort is put into the development of low-dose CBCT protocols, and many manufacturers already offer an indicationdependent pre-programmed FOV and imaging parameters as an option. Yet, there is no specific FOV size for imaging impacted canines.

The smaller the FOV, the higher the risk for structures falling outside the final image. Utilizing scout views and using light beam markers or laser lights coinciding with the patient's anatomic landmarks in patient positioning guarantee that structures of interest locate inside the tomographic volume [1].

As a step toward development of a canine-specific CBCT program, the present study aims at defining the minimum size of FOV, capable of encompassing the impacted or unerupted maxillary canines, and determining its relation to anatomic landmarks.

\section{Materials and methods}

\section{The radiographic sample}

The retrospective study material consisted of 188 consecutive CBCT scans of Finnish children and adolescents (aged $<17$ years), who had been referred for CBCT between December 2007 and October 2012 at the Department of Oral and Maxillofacial Diseases, University of Helsinki, Finland. The study material did not contain patients fulfilling the criteria for special hospital health care and treated in a hospital setting, such as patients with cleft palate or other craniofacial deformity. For the present study, we selected the scans where the indication for imaging was unilateral or bilateral impaction or delayed eruption of a maxillary canine $(N=71)$.

The CBCT scans had been taken with Promax 3D (Planmeca, Finland). Different FOV sizes had been used in the different scans: $4 \times 5 \mathrm{~cm}(N=32), 6 \times 6 \mathrm{~cm}(N=7)$, $8 \times 5 \mathrm{~cm}(N=23)$, and $8 \times 8 \mathrm{~cm}(N=9)$. Voxel size in all CBCT scans was $200 \mu \mathrm{m}$ or smaller. All radiographic data were analyzed with Romexis ${ }^{\circledR}$ software (Planmeca, Helsinki, Finland) and Barco MDCG-212 grayscale display (Barco, Kortrijk, Belgium) with optimal ambient lighting conditions by a dentist who had acquired additional competency in CBCT examinations (A.-M.I.). In unclear cases, senior oral radiologists (E.P.E. and M.E.) were consulted.

Permission to re-examine the CBCT data and patient files were received from the Oral Healthcare Department of City of Helsinki (HEL 2012-005583) and from the University of Helsinki.

\section{Canine development}

To assure that the assessed minimum size of FOV was not underestimated due to underdevelopment of the canine roots, the developmental status of the canines was evaluated according to Demirjian et al. (1973) [10]. The method divides permanent tooth development into eight stages, A-H. Shortly, stages A-D include development of the crown from onset of mineralization to completion of the cementoenamel junction. At stage E, pulp horn is visible and root length is still less than crown height. At stage $\mathrm{F}$, walls of the pulp chamber form an isoscele triangle and root length is equal or more than crown height. At stage $\mathrm{G}$, walls of the root canal are parallel and full root length has been achieved. Apex is open. Stage $\mathrm{H}$ includes teeth with closed apex and continuous periodontal ligament.

\section{Canine localization and optimization of the FOV}

The CBCT scans were then examined.

1. To locate the impacted canines in an X-Y-Z-coordinate defined by three anatomical reference planes that were placed in the 3D view at right angles in relation to each other:

(a) Midsagittal plane; set along the intermaxillary suture (IS) from the anterior nasal spine (ANS) to the posterior nasal spine (PNS)

(b) Horizontal plane; set at the level of the hard palate from ANS to PNS

(c) Frontal plane; set at the most labial point of the labial surface of the maxillary central incisors 
2. To define the smallest 3D size of a cylinder that accommodates all the located canines separately on the right and on the left side

3. To define the position of that cylinder, to optimize the placement of the tomographic volume

The CBCT scans were adjusted by the best fit by aboveset reference planes. The perpendicular minimum and maximum distances between any part of each canine and the three reference planes were assessed as follows:

1. The distance of the most medial part of canine from the midsagittal plane (Fig. 1a)

2. The distance of the most lateral part of canine from the midsagittal plane (Fig. 1b)

3. The distance of the most inferior part of canine from the horizontal plane (Fig. 2a)

4. The distance of the most superior part of canine from the horizontal plane (Fig. 2b)

5. The distance of the most anterior part of canine from the frontal plane (Fig. 3a)

6. The distance of the most posterior part of canine from the frontal plane (Fig. 3b)

\section{Statistical analyses}

Intra-examiner repeatability was tested by calculating the range of error, mean error, and random error using the "method of moments" estimator with $95 \%$ confidence interval between replicated measurements [11]. Two-tailed $t$ test was performed in Microsoft Excel (Microsoft Corporation, Redmond, WA, USA) to analyze variation between maxillary right and left canine positions, and between intra-examiner repeatability of cranio-caudal, medio-lateral, and anteroposterior measurements.

\section{Results}

\section{Final sample}

Analysis of the 71 CBCT scans showed that the planned measurements could not be performed on 28 because there were artifacts $(N=3)$, the canine fell partly outside the FOV $(N=2)$, or the anatomic structures needed to construct the $3 \mathrm{D}$ coordinate-labial surfaces of maxillary incisors in the majority of cases-were not visible in the FOV $(N=23)$. All different FOV sizes were represented in the groups of both excluded and included scans. The FOV sizes of the final sample were $4 \times 5 \mathrm{~cm}(N=16), 6 \times 6 \mathrm{~cm}(\mathrm{~N}=2), 8 \times 5 \mathrm{~cm}$ $(N=17)$, and $8 \times 8 \mathrm{~cm}(N=8)$. The final sample comprised $43 \mathrm{CBCT}$ scans of 50 maxillary impacted canines (24 rightside and 26 left-side canines) of 43 patients. Of the patients, 31 were female and 12 were male, and the age range was 9.7 to 16.0 years with a mean age of 12.8 years.

\section{Developmental stages of impacted canines}

All canines represented Demirjian stages F, G, or H. On the right, stage- $F$ canines were recorded in two females aged 10.6 and 11.4 years. Stage $\mathrm{G}$ was recorded in 9 teeth, and stage $\mathrm{H}$ in 11. Similar on the left, stage-F canines were seen in three females aged 9.7 to 11.5 years. Stage $\mathrm{G}$ was recorded in 11 teeth, and stage $\mathrm{H}$ in 12 . Hence, $92 \%$ of the
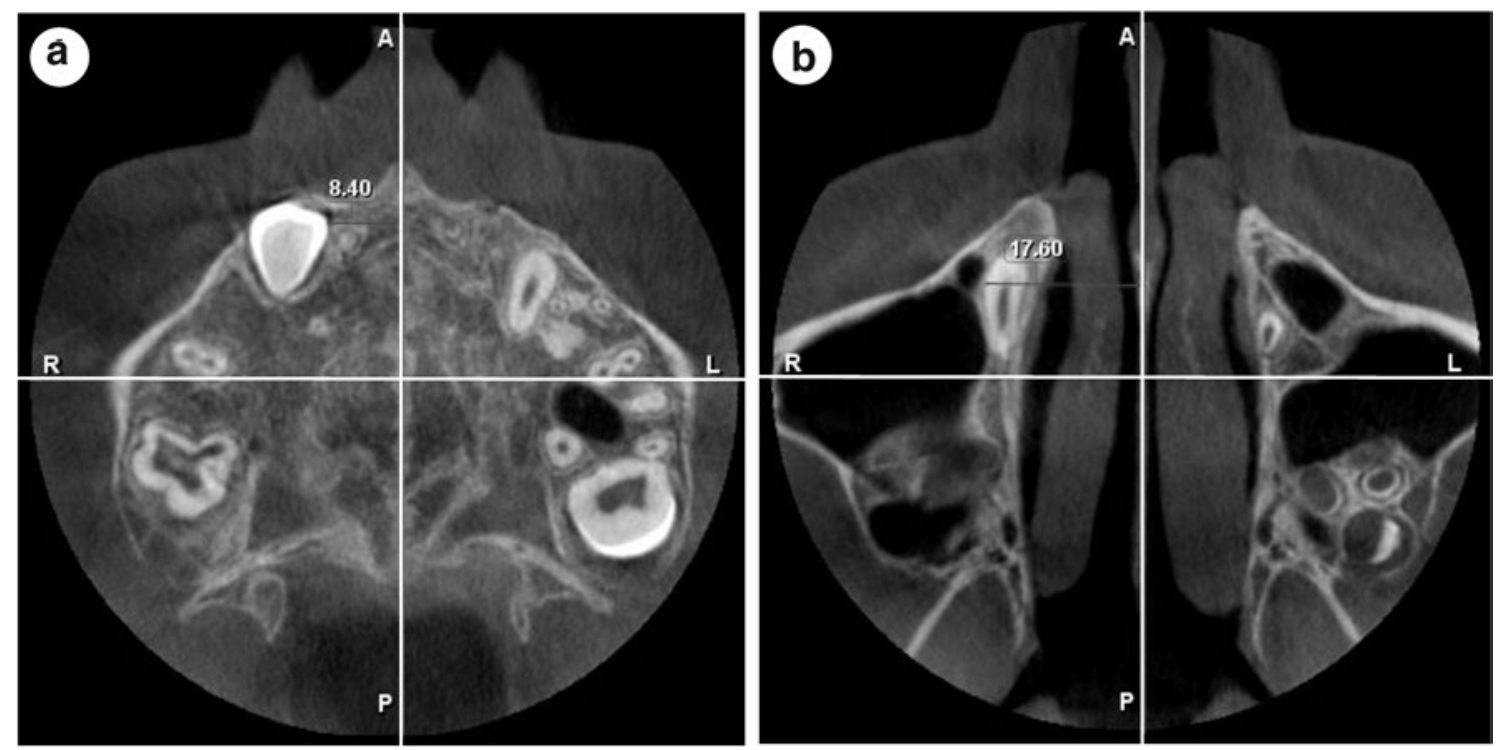

Fig. 1 CBCT image, axial view, illustrating the perpendicular measurements between a the most medial and $\mathbf{b}$ the most lateral part of an impacted canine and the midsagittal reference plane, set along the intermaxillary suture from the anterior nasal spine to the posterior nasal spine 


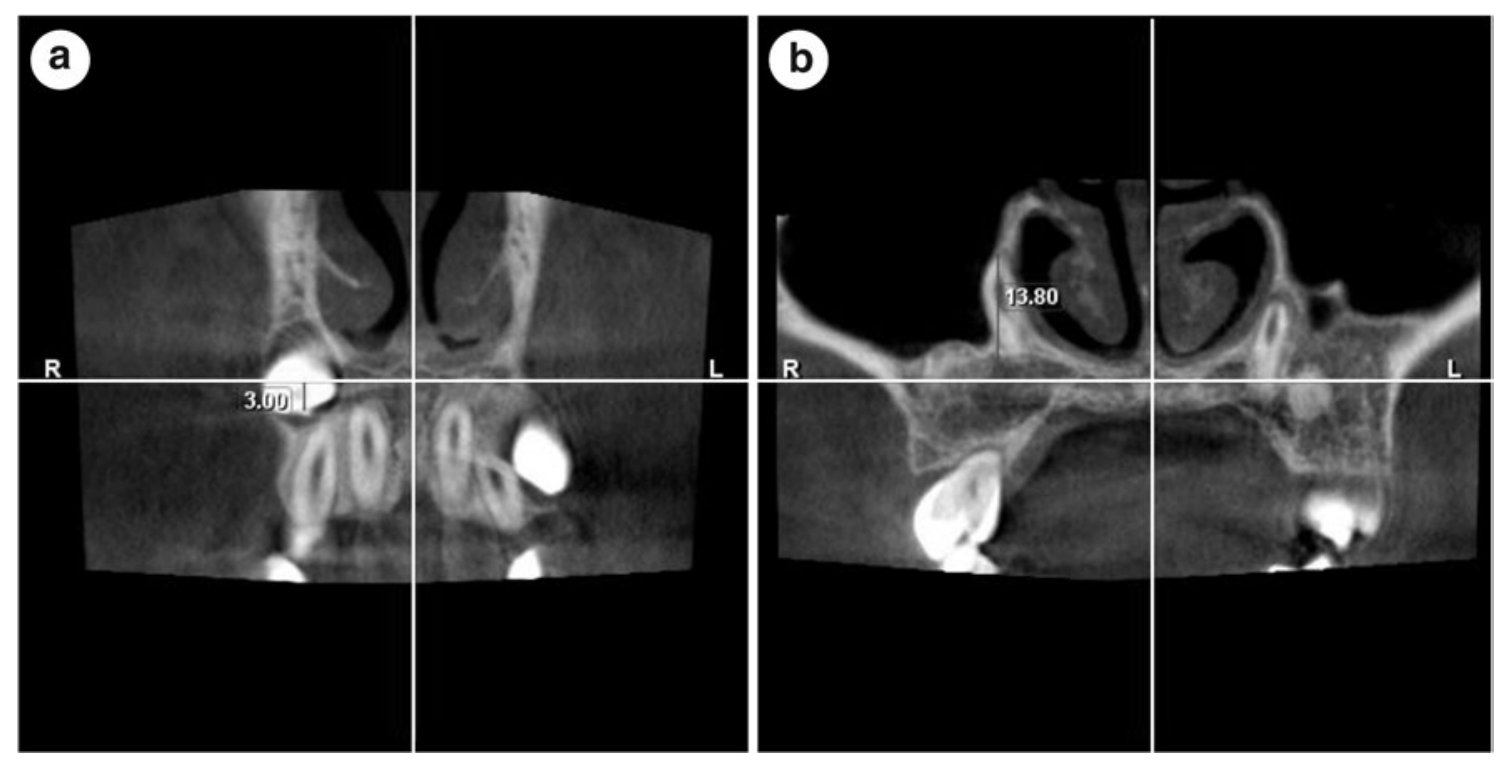

Fig. 2 CBCT image, coronal view, illustrating the perpendicular measurements between $\mathbf{a}$ the most inferior and $\mathbf{b}$ the most superior part of an impacted canine and the horizontal reference plane, set across hard palate from the anterior nasal spine to the posterior nasal spine

right-side and $88 \%$ of the left-side canines had achieved a full root length (stages $\mathrm{G}$ and $\mathrm{H}$ ).

\section{Location of impacted canines}

In cranio-caudal direction The most superior part of any of the maxillary right canines was situated at maximum $13.8 \mathrm{~mm}$ above the level of the hard palate, and the most inferior part at maximum $18.1 \mathrm{~mm}$ below the level of the hard palate (Fig. 4). The most superior part of any of the maxillary left canines was situated at maximum $10.6 \mathrm{~mm}$ above the level of the hard palate, and the most inferior part at maximum $19.4 \mathrm{~mm}$ below the level of the hard palate (Fig. 5).

In medio-lateral direction The most medial part of any maxillary right canine was situated at closest $1.2 \mathrm{~mm}$ from the midsagittal plane, and the most lateral part at furthest $20.5 \mathrm{~mm}$ from it. The most medial one of the maxillary left canines crossed the midsagittal plane by $1.9 \mathrm{~mm}$, and the most lateral part of any left canine was situated at furthest $21.2 \mathrm{~mm}$ from it (Fig. 6).

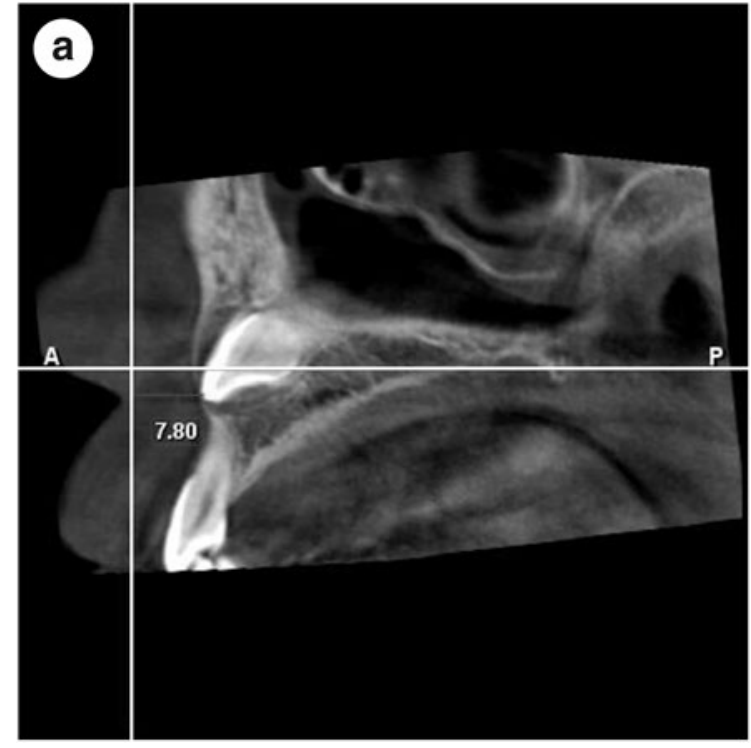

Fig. 3 CBCT image, sagittal view, illustrating the perpendicular measurements between $\mathbf{a}$ the most anterior and $\mathbf{b}$ the most posterior part of an impacted canine and the frontal plane, set at the most labial

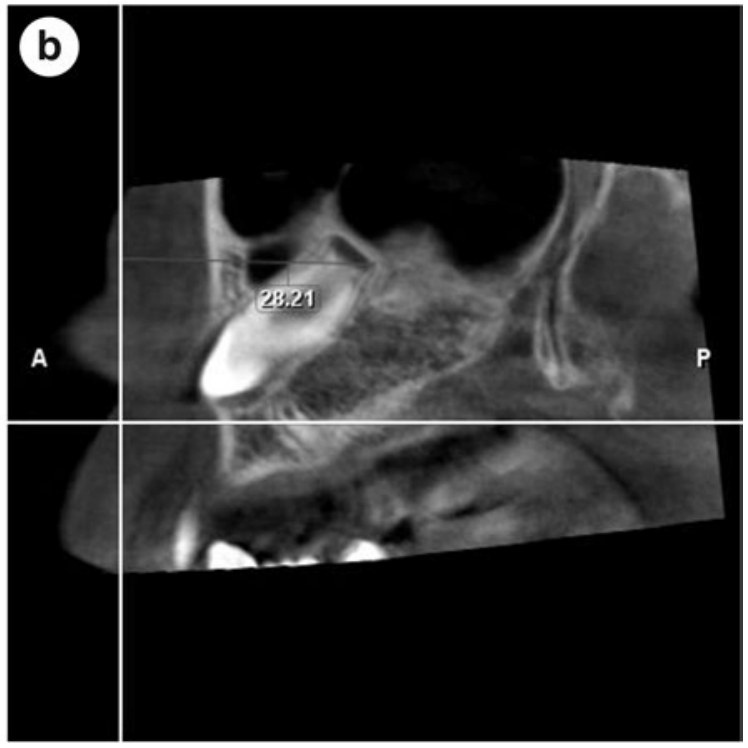

point of the labial surface of the maxillary central incisors at a right angle to the horizontal plane 


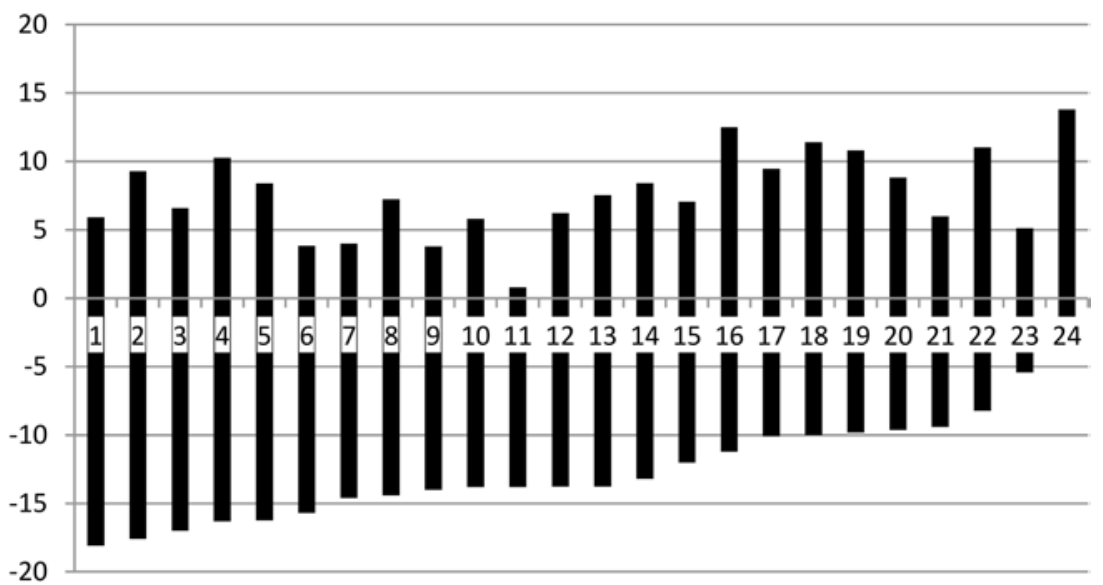

Fig. 4 Vertical position of the impacted maxillary right canines, numbered from 1 to 24 . The graph shows their maximum superior and inferior distances $(\mathrm{mm})$ in relation to the horizontal reference plane

In antero-posterior direction Any part of the maxillary, right canines were situated 3.0 to $30.9 \mathrm{~mm}$ behind the frontal plane set at the level of the labial surfaces of maxillary central incisors. The respective range on the left was 2.9 to $33.3 \mathrm{~mm}$ (Fig. 6).

\section{Size of the FOV}

The height of a cylinder that would completely accommodate all maxillary right canines, despite of their position, was calculated as $31.9 \mathrm{~mm}$ and its diameter as $30.0 \mathrm{~mm}$, whereas in the left, the height of the cylinder would be $30.0 \mathrm{~mm}$ and its diameter $39.0 \mathrm{~mm}$ (Figs. 4, 5, and 6).

\section{Position of FOV}

To completely accommodate all maxillary right canines in the present sample the top of the above-sized cylinder (level 0), set at the level of the hard palate. The length of each bar reflects the combined effect of the actual length of the tooth and its verticality

would situate $13.8 \mathrm{~mm}$ above the hard palate, its medial edge $3.4 \mathrm{~mm}$ across the midline, and anterior edge $2.4 \mathrm{~mm}$ behind the labial surface of maxillary central incisors. For the left-side canines, the respective measures were top of the volume $10.6 \mathrm{~mm}$ above the hard palate, medial edge $8.4 \mathrm{~mm}$ across the midline, and anterior edge $2.5 \mathrm{~mm}$ in front of the labial surface of maxillary central incisors (Figs. 4, 5, and 6).

\section{Combined results for right- and left-side canines}

The variation in the position between maxillary right and left canines was not statistically significant in any direction-medial, lateral, anterior, posterior, inferior, or superior $(0.12 \leq P \leq 0.86)$. Combining the results, the minimum FOV would be $3.9(D \varnothing) \times 3.3(H) \mathrm{cm}$, with its top situated $13.8 \mathrm{~mm}$ above the hard palate, its medial edge $8.4 \mathrm{~mm}$ across the midline, and anterior edge $2.5 \mathrm{~mm}$ in front of the labial surface of maxillary central incisors.

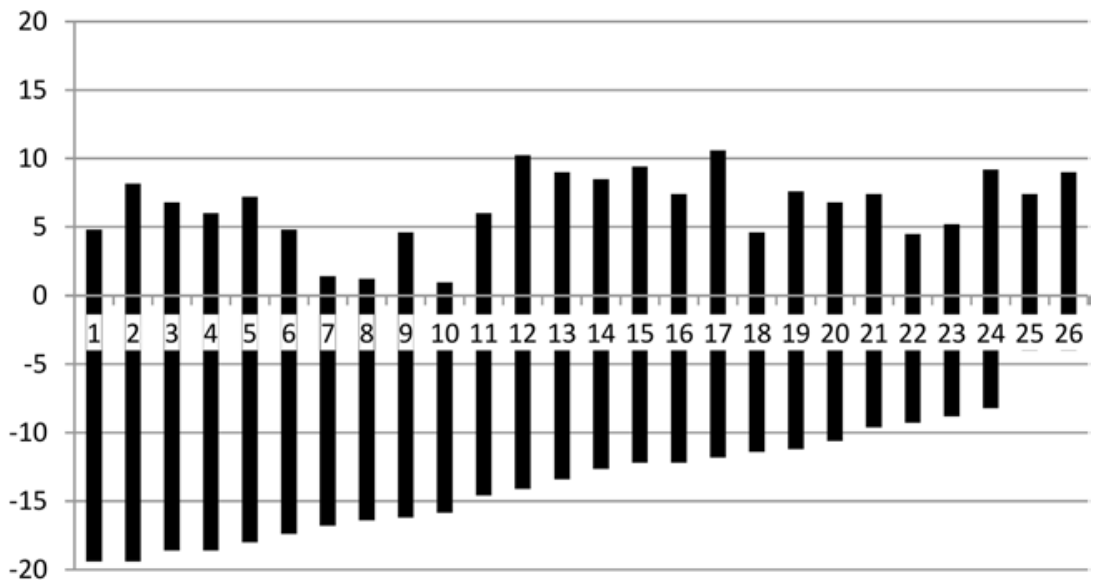

Fig. 5 Vertical position of the impacted maxillary left canines, numbered from 1 to 26 . The graph shows their maximum superior and inferior distances $(\mathrm{mm})$ in relation to the horizontal reference plane (level 0), set at the level of the hard palate. The length of each bar reflects the combined effect of the actual length of the tooth and its verticality 


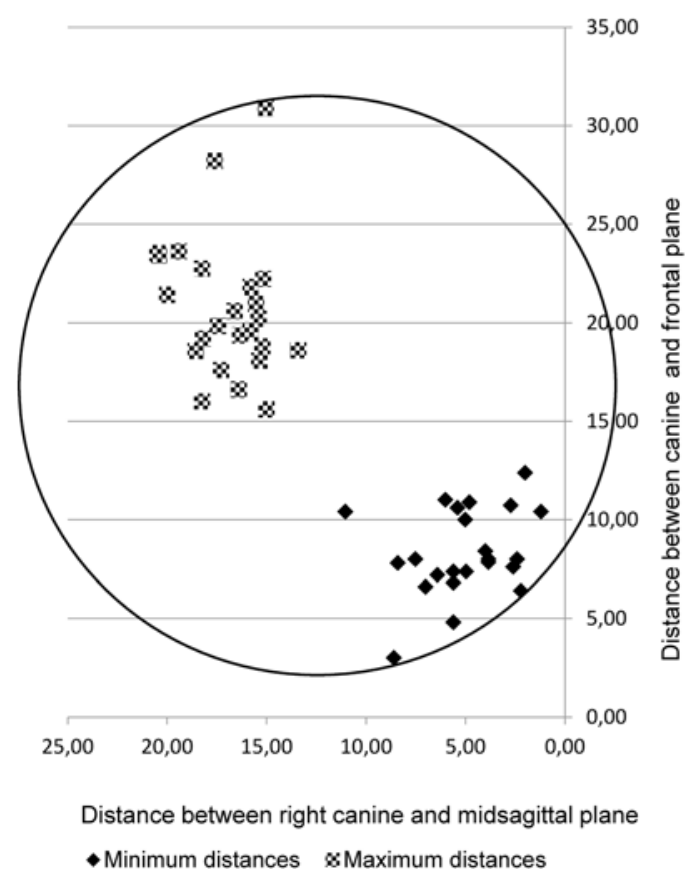

Fig. 6 Horizontal position of the impacted 24 maxillary right and 26 maxillary left canines. The two-dimensional graph shows their minimum and maximum medio-lateral and antero-posterior distances $(\mathrm{mm})$ from the reference planes (levels 0), the midsagittal plane set

\section{Pathological findings}

Pathological cystic or inflammatory lesions were not diagnosed in association with any of the canines.

\section{Intra-examiner repeatability}

Replicated analyses were performed after 12 months on a random sample of $15 \mathrm{CBCT}$ scans, where the original settings had been restored. This means that all three reference planes were set again, and new measurements were made, six for each impacted canine. The mean random errors ranged between $0.13 \mathrm{~mm}$ and $0.34 \mathrm{~mm}$ (Table 1). There were no statistically significant differences between repeatability of results in medio-lateral, cranio-caudal, and anteroposterior directions $(0.08 \leq P \leq 0.92)$.

\section{Discussion}

Presumably, in the nearest future, CBCT scans extensively replace conventional 2D methods with respect to localization of unerupted and supernumerary teeth [12]. Therefore, optimization of the method for dose reduction is a timely issue particularly in children that possess a higher attributable lifetime radiation-related mortality risk than adults from CBCT scans [13].

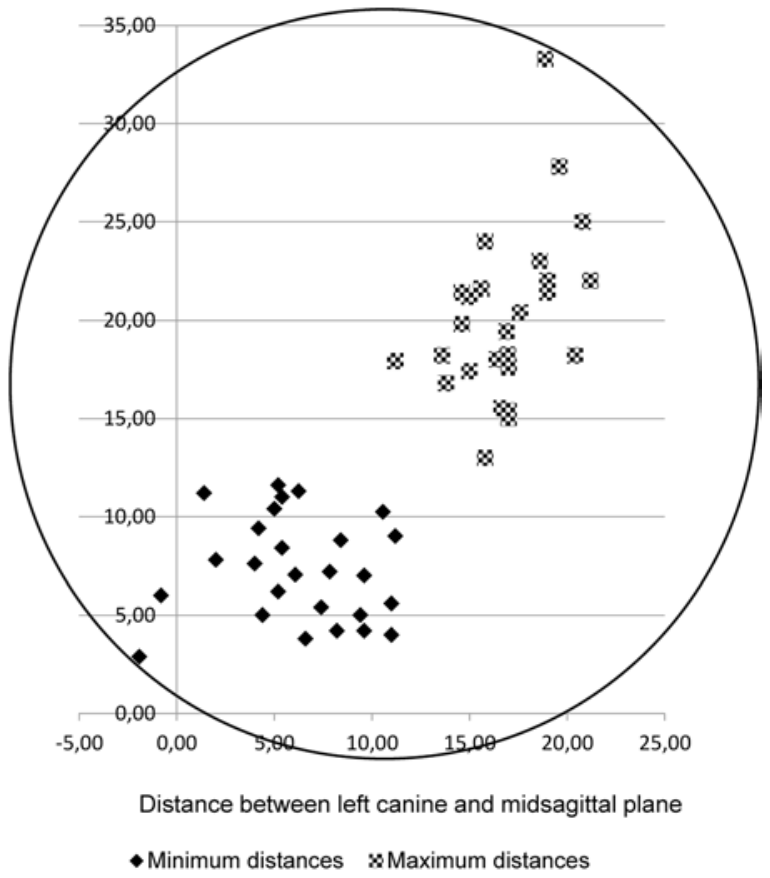

along the intermaxillary suture, and the frontal plane set at the most labial point of the maxillary central incisors. The circles indicate the smallest diameter and optimum horizontal positioning of vertical cylinders that accommodate the canines

Based on phantom studies, CBCT scans of a 10-yearold result in 15 to 140 times higher organ doses compared to conventional dental radiography [14], and the received equivalent dose is significantly higher in children than in adults during CBCT examinations especially in maxillary regions $[15,16]$. Based on dosimetry of patients during CBCT, and conversion of skin doses to organ dose, the lifetime attributed cancer risk has been estimated as 9.8 per million for 8- to 11-year-olds and 2.7 per million for older than 60 years, the risk being $40 \%$ higher for females [17]. Risk on cancer has been estimated as 0.3 to $1.3 \times 10^{-6}$ for dental panoramic tomography, as $0.2 \times 10^{-6}$ for cephalometric radiography, and as $61.5 \times 10^{-6}$ for CBCT with a large FOV [18]. A large FOV increases the dose for sensitive organs such as the brain and the thyroid gland [19]. Notably the radiation dose in DPTs taken with different devices is within the same order of magnitude, whereas the use of different CBCT devices and their different scanning programs result in widely variable effective doses [19-21].

Several studies emphasize the importance of optimizing imaging parameters and collimation of beam in CBCT scans $[13,22]$, but studies offering protocols for further optimization of CBCTs in children are rare in the literature. In a child phantom study focusing on optimization of CBCT scans of impacted maxillary canines with a small $(4 \times 4 \mathrm{~cm}) \mathrm{FOV}$, reduction of tube voltage and current from the recommendations of the manufacturer resulted in a 50\% dose reduction, 
Table 1 Intra-observer repeatability of the measurements from 15 CBCT scans

\begin{tabular}{|c|c|c|c|c|c|c|}
\hline \multirow[t]{2}{*}{$\begin{array}{l}\text { Difference between repeated } \\
\text { measurements }(\mathrm{mm})\end{array}$} & \multicolumn{2}{|c|}{$\begin{array}{l}\text { Distance of canine from the } \\
\text { medio-lateral reference plane }\end{array}$} & \multicolumn{2}{|c|}{$\begin{array}{l}\text { Distance of canine from the cranio- } \\
\text { caudal reference plane }\end{array}$} & \multicolumn{2}{|c|}{$\begin{array}{l}\text { Distance of canine from the antero- } \\
\text { posterior reference plane }\end{array}$} \\
\hline & Minimum & Maximum & Minimum & Maximum & Minimum & Maximum \\
\hline Range & $0.00-0.32$ & $0.00-1.20$ & $0.00-0.80$ & $0.00-0.80$ & $0.00-0.80$ & $0.00-1.40$ \\
\hline Mean & 0.05 & 0.15 & 0.02 & 0.10 & 0.12 & 0.33 \\
\hline Random error & 0.13 & 0.33 & 0.29 & 0.31 & 0.19 & 0.34 \\
\hline $95 \% \mathrm{CI}^{\mathrm{a}}$ of random error & $0.07-0.19$ & $0.17-0.49$ & $0.14-0.43$ & $0.16-0.46$ & $0.10-0.29$ & $0.17-0.51$ \\
\hline
\end{tabular}

${ }^{\mathrm{a}}$ Confidence interval

an acceptable image quality retained [23]. Reduction of FOV size has proven even more significant in the reduction of effective dose in children than in adults $[13,20]$, and the use of thyroid shield is recommended [24, 25]. While it has also been recommended to reduce the beam height when imaging impacted canines, a suggested FOV size has not been specified [18].

In the present study, we measured from $50 \mathrm{CBCT}$ scanned impacted maxillary canines that the minimum FOVs, covering all maxillary right and left canines, were $3.0 \varnothing \times 3.2 \mathrm{~cm}$ and $3.9 \varnothing \times 3.0 \mathrm{~cm}$, respectively. The result reflects how outliers increase the size demand of the FOV. Because of the cylindrical form, a mediolaterally or anterior-posteriorly deviating object has a relatively greater effect on FOV than a vertically dislocated one. Most probably, by increasing the sample, the positions of the canines would become even more dispersed, indicating the need of a larger FOV, and the relatively small sample size forms a limitation of the study. With few exceptions, canines are, however, likely to be optimally scanned using a small FOV, such as $4.0 \varnothing \times 3.5 \mathrm{~cm}$. A field of this size and positioned slightly across the midline, as defined in this paper, will also include the roots of the incisors that are in risk of associated root resorption and of interest for the clinician. Regarding the midline, several papers report on transmigration of impacted mandibular canines, whereas the phenomenon is rare in the maxilla [26]. It was interesting to find in the present sample that two of the left-side canines partly traversed the midsagittal facial plane (Fig. 6).

Comparison to previous studies is hampered by differences in the methodology. Most studies concentrate on the relative efficacy of CBCT in comparison to $2 \mathrm{D}$ imaging in localization of the canines as palatal or buccal, or on diagnostic accuracy of adjacent tooth root resorption. Comparable precise 3D data on canine location could not be found, and identical reference lines have not been used. The repeatability of the reference line placement and measurements in the present study was excellent with clinically non-significant mean and random errors, exhibiting a maximum $95 \%$ upper confidence interval limit of $0.51 \mathrm{~mm}$.
Moreover, mere localization of tooth root apeces and incisal tips, common for canine studies, do not fully serve the purpose of evaluation of optimum FOV volume, since the tooth is not a narrow bar but a notably spindle-shaped structure. Another rarely studied feature in canine studies is the eventual completion of root length development. Here, most canines had reached full root length, justifying the suggested FOV. Finally, in most studies, right- and left-side data have been grouped. Human faces are not symmetrical. Several studies [27] report the frequent dominance of the left side of the face both in healthy males and females. Therefore, symmetry in the canine impaction pattern could not be taken as a standpoint in the analysis. It turned out that indeed the left side showed more variation in the canine position, even though statistically significant differences were not found in this 50-tooth sample. Our observation that the general pattern of impaction of right- and left-side canines may not necessarily be identical deserves to be studied more closely.

The other objective of our study was to analyze the proper placement of the FOV. Placing a very small tomographic volume to the area of impacted teeth that are not clinically visible is challenging and not comparable to situation in phantom skull studies [23]. Therefore, a minimum FOV protocol may not be suitable for imaging impacted maxillary teeth, unless there are clinical landmarks to facilitate FOV placement. In addition, radiographers should be skilled and experienced enough to be able to place small FOVs into the proper position to prevent cutting off structures and repeated exposures. Knowing the size of the indication-dependent FOVs allows design of programs for automatic FOV placement through control panel of the device. Possible positioning errors could be fixed and adjusted according to 2D-scout views before the final exposure. Another alternative, proven equally good for accurate patient positioning, is the use of a specific aligner placed into the patient's oral cavity [28]. The choice of labial surface of maxillary incisors as a reference structure was to serve the clinician an easily defined landmark. It can be criticized that this landmark shows high variation between patients due to differences in incisor position and inclination. This probably also applies to our study 
group, although not analyzed. Moreover, from a methodological point of view, the landmark selection reduced the number of CBCT scans applicable for the present analysis.

For selection of accurate FOV size and its optimum positioning, information on impacted canines obtained from a DPT or lateral and posteroanterior scout views prior to the actual CBCT scanning are essential. The criteria of a good periapical radiograph list that $2 \mathrm{~mm}$ of surrounding bone structure should be visible [29]. Similar criteria, to our knowledge, have not been listed regarding dental CBCT. Since CBCT is taken not only for diagnostic purposes, such as assessment of exact location of the tooth, its morphology, and intact root surface, but also to support the treatment plan and facilitate safe surgical exposure, removal, or transplantation of the tooth or its successful orthodontic traction. Since these are performed in an area that may contain vital adjacent anatomical structures such as floor of the maxillary sinus, floor and lateral wall of the nasal cavity, incisive canal, and adjacent teeth, we suggest a view extended by $5 \mathrm{~mm}$ in each direction. Even taking this into account, our study indicates that the adequate FOV for imaging of maxillary impacted canines in children could be smaller than the fixed minimum FOV of many popular CBCT-devices [19]. The same concern exists for endodontic applications.

It is vital that the optimization of CBCT, like all other methods exposing the patients to ionizing radiation, takes place after the justification processes. CBCTs are only justified when true indication exists [1]. Today, the eventual indication of CBCT for imaging of impacted canines rises after 2D imaging, most often DPT, in cases where conventional radiography does not provide enough information for diagnosis and decision making and for surgical and/or orthodontic procedures to be carried out safely. Often, the question is about exact position of the canine in relation to other teeth and other anatomic structures and of eventual resorptions of close-by roots. Preliminary estimation of the position of the canines on an existing DPT before the CBCT scan gives valuable tools for decision making of whether a reducedsized FOV can be applied. This requires further studies on selection of positional-dependent size for FOV. Other areas of future research are dose reduction achieved by using the suggested FOVs compared to standard FOVs, as well as clinical experimenting with smaller FOVs to identify pitfalls in the clinical practice. This, however, would require cooperation with manufacturers.

To conclude, it is important to reduce the FOV in children's CBCT for radiation protection. For imaging of maxillary impacted canines, a $4 \varnothing \times 3.5 \mathrm{~cm}$ volume is generally large enough, provided it can be positioned optimally. Automated patient positioning and scout views would be means to decrease the threshold of applying small FOVs.
Funding Open access funding provided by University of Helsinki including Helsinki University Central Hospital. The work was supported by a grant from Finnish Women Dentists to Anne-Mari Ilo.

\section{Compliance with ethical standards}

Conflict of interest The authors declare that they have no conflict of interest.

Ethical approval All procedures performed in the study were in accordance with the ethical standards of the institutional and/or national research committee and with the 1964 Helsinki declaration and its later amendments or comparable ethical standards.

Informed consent For this type of study, formal consent is not required.

Open Access This article is distributed under the terms of the Creative Commons Attribution 4.0 International License (http://creativecommons.org/licenses/by/4.0/), which permits unrestricted use, distribution, and reproduction in any medium, provided you give appropriate credit to the original author(s) and the source, provide a link to the Creative Commons license, and indicate if changes were made.

\section{References}

1. European Commission (2012) Radiation protection no 172. Cone beam CT for dental and maxillofacial radiology. Evidence-based guidelines. Luxemburg: SEDENTEXCT. http://www.sedentexct. eu/files/radiation_protection_172.pdf. Accessed 1 Nov 2018

2. Hidalgo-Rivas JA, Theodorakou C, Carmichael F, Murray B, Payne M, Horner K (2014) Use of cone beam CT in children and young people in three United Kingdom dental hospitals. Int J Paediatr Dent 24:336-348

3. Isman Ö, Yilmaz HH, Aktan AM, Yilmaz B (2017) Indication for cone beam computed tomography in children and young patients in a Turkish subpopulation. Int J Paediatr Dent 27:183-190. https://doi.org/10.1111/ipd.12250

4. Haney E, Gansky SA, Lee JS, Johnson E, Maki K, Miller AJ, Huang JC (2010) Comparative analysis of traditional radiographs and cone-beam computed tomography volumetric images in the diagnosis and treatment planning of maxillary impacted canines. Am J Orthod Dentofac Orthop 137:590-597. https://doi. org/10.1016/j.ajodo.2008.06.035

5. Botticelli S, Verna C, Cattaneo PM, Heidmann J, Melsen B (2011) Two- versus three-dimensional imaging in subjects with unerupted maxillary canines. Eur J Orthod 33:344-349. https:// doi.org/10.1093/ejo/cjq102

6. Algerban A, Jacobs R, Fieuws S, Willems G (2011) Comparison of two cone beam computed tomographic systems versus panoramic imaging for localization of impacted maxillary canines and detection of root resorption. Eur J Orthod 33:93-102. https://doi. org/10.1093/ejo/cjq034

7. Lai CS, Bornstein MM, Mock L, Heuberger BM, Dietrich T, Katsaros C (2013) Impacted maxillary canines and root resorptions of neighbouring teeth: a radiographic analysis using conebeam computed tomography. Eur J Orthod 35:529-538. https:// doi.org/10.1093/ejo/cjs037

8. Wriedt S, Jaklin J, Al-Nawas B, Wehrbein H (2012) Impacted upper canines: examination and treatment proposal based on 3D versus 2D diagnosis. J Orofac Orthop 73:28-40. https://doi. org/10.1007/s00056-011-0058-8 
9. Oenning AC, Jacobs R, Pauwels R, Stratis A, Hedesiu M, Salmon B, DIMITRA Research Group, http://www.dimitra.be (2018) Cone-beam CT in paediatric dentistry: DIMITRA project position statement. Pediatr Radiol 48: 308-316. https://doi.org/10.1007/ s00247-017-4012-9

10. Demirjian A, Goldstein H, Tanner JM (1973) A new system of dental age assessment. Hum Biol 45:211-227

11. Springate SD (2012) The effect of sample size and bias on the reliability of estimates of error: a comparative study of Dahlberg's formula. Eur J Orthod 34:158-163. https://doi.org/10.1093/ejo/ cjr010

12. Aps JK (2013) Cone beam computed tomography in paediatric dentistry: overview of recent literature. Eur Arch Paediatr Dent 14:131-140. https://doi.org/10.1007/s40368-013-0029-4

13. Theodorakou C, Walker A, Horner K, Pauwels R, Bogaerts R, Jacobs, SEDENTEXCT Project Consortium (2012) Estimation of paediatric organ and effective doses from dental cone beam CT using anthropomorphic phantoms. Br J Radiol 85:153-160. https://doi.org/10.1259/bjr/19389412

14. Kadesjö N, Lynds R, Nilsson M, Shi XQ (2018) Radiation dose from X-ray examinations of impacted canines: cone beam CT vs two-dimensional imaging. Dentomaxillofac Radiol 47:20170305. https://doi.org/10.1259/dmfr.20170305

15. Yepes JF, Booe MR, Sanders BJ, Jones JE, Ehrlich Y, Ludlow JB, Johnson B (2017) Pediatric phantom dosimetry of Kodak 9000 cone-beam computed tomography. Pediatr Dent 39:229-232

16. Al Najjar A, Colosi D, Dauer LT, Prins R, Patchell G, Branets I et al (2013) Comparison of adult and child radiation equivalent doses from 2 dental cone-beam computed tomography units. Am J Orthod Dentofac Orthop 143:784-792. https://doi.org/10.1016/j. ajodo.2013.01.013

17. Pauwels R, Cockmartin L, Ivanauskaite D, Urboniene A, Gavala S, Donta C et al (2014) Estimating cancer risk from dental conebeam CT exposures based on skin dosimetry. Phys Med Biol 59:3877-3891. https://doi.org/10.1088/0031-9155/59/14/3877

18. Brooks SL (2009) CBCT dosimetry: orthodontic considerations. Semin Orthod 15:14-18. https://doi.org/10.1053/j. sodo.2008.09.002

19. Marcu M, Hedesiu M, Salmon B, Pauwels R, Stratis A, Oenning ACC, Cohen ME, Jacobs R, Baciut M, Roman R, Dinu C, Rotaru H, Barbur I, DIMITRA Research Group (2018) Estimation of the radiation dose for pediatric $\mathrm{CBCT}$ indications: a prospective study on ProMax3D. Int J Paediatr Dent 28:300-309. https://doi. org/10.1111/ipd.12355

20. Batista WO, Navarro MV, Maia AF (2012) Effective doses in panoramic images from conventional and CBCT equipment. Radiat Prot Dosim 151:67-75. https://doi.org/10.1093/rpd/ncr454

21. Al-Okshi A, Lindh C, Salé H, Gunnarsson M, Rohlin M (2015) Effective dose of cone beam CT (CBCT) of the facial skeleton: a systematic review. Br J Radiol 88:20140658. https://doi. org/10.1259/bjr.20140658

22. Jacobs R, Quirynen M (2014) Dental cone beam computed tomography: justification for use in planning oral implant placement. Periodontol 2000 66:203-213. https://doi.org/10.1111/prd.12051

23. Hidalgo Rivas JA, Horner K, Thiruvenkatachari B, Davies J, Teodorakou C (2015) Development of a low-dose protocol for cone beam CT examinations of the anterior maxilla in children. Br J Radiol 88:20150559. https://doi.org/10.1259/bjr.20150559

24. Hidalgo A, Davies J, Horner K, Theodorakou C (2015) Effectiveness of thyroid gland shielding in dental CBCT using a paediatric anthropomorphic phantom. Dentomaxillofac Radiol 44:201402285. https://doi.org/10.1259/dmfr.20140285

25. Signorelli L, Patcas R, Peltomäki T, Schätzle M (2016) Radiation dose of cone-beam computed tomography compared to conventional radiographs in orthodontics. J Orofac Orthop 77:9-15. https://doi.org/10.1007/s00056-015-0002-4

26. Aydin U, Yilmaz HH (2003) Transmigration of impacted canines. Dentomaxillofac Radiol 32:198-200. https://doi.org/10.1259/ $\mathrm{dmfr} / 38819077$

27. Ercan I, Ozdemir ST, Etoz A, Sigirli D, Tubbs RS, Loukas M, Guney I (2008) Facial asymmetry in young healthy subjects evaluated by statistical shape analysis. J Anat 213:663-669. https://doi. org/10.1111/j.1469-7580.2008.01002.x

28. Dawood A, Sauret-Jackson V, Patel S, Darwood A (2010) A novel alignment device for cone beam computed tomography: principle and application. Dentomaxillofac Radiol 39:375-382. https://doi. org/10.1259/dmfr/21679313

29. European Commission (2004) European Guidelines on Radiation Protection in Dental Radiology. The Safe Use of Radiographs in Dental Practice. EC report 136, Brusseles, Belgium. https://ec.europa. eu/energy/sites/ener/files/documents/136.pdf. Accessed 3 Feb 2019

Publisher's note Springer Nature remains neutral with regard to jurisdictional claims in published maps and institutional affiliations. 